\title{
Numerical and experimental study of the time-dependent states and the slow dynamics in a von Kármán swirling flow
}

\author{
E. CRESPO DEL ARCO*†, J. J. SÁNCHEZ-ÁLVAREZł, \\ E. SERRE $\S$, A. DE LA TORRE $\uparrow$ and J. BURGUETE \\ $†$ Departamento de Física Fundamental, U.N.E.D., Madrid, 28080, Spain \\ \$E.T.S.I. Aeronáuticos, Universidad Politécnica de Madrid, Madrid, 28040, Spain \\ §M2P2 UMR6181 CNRS/Universités d'Aix-Marseille, Marseille, 13451, France \\ - Departamento de Física y Matemática Aplicada, Universidad de Navarra, \\ E-31080, Pamplona, Spain
}

(Received 7 February 2008; in final form 1 October 2008)

\begin{abstract}
The characteristics of time-dependent swirling flows in a von Kármán configuration are investigated numerically up to Reynolds number $\mathrm{Re}=3000$ (based on the angular velocity and the radius of the cylinder), and experimentally within turbulent regimes. Experimental results are analyzed together with the periodic and aperiodic flow obtained numerically. In the present configuration, the fluid is contained in a cylindrical cavity with aspect ratio (height to radius) $\Gamma=2$ and the motion is driven by the exact counter rotation of the end walls while the sidewall is at rest. Spectral direct numerical simulations show that for this geometry the axisymmetric base flow becomes unstable to non-axisymmetric perturbations with azimuthal wavenumber $m=1$ through a subcritical bifurcation, and the corresponding flow exhibits a pattern with one cat's eye in the axial-azimuthal planes. Increasing the Reynolds number the flow becomes unstable to non-axisymmetric steady perturbations with even azimuthal wavenumbers, and the corresponding flows exhibits a two cat's eyes pattern. The occurrence of cat's eye pattern in radial-azimuthal surfaces was observed in this and other aspect ratio cavities and is associated with vortices in 3D steady flows with characteristic azimuthal modes [Nore, C., Tuckerman, L.S., Daube, O. and Xin, S., The $1: 2$ mode interaction in exactly counter-rotating von Kármán swirling flow, J. Fluid Mech., 2003, 477, 51-88; Lackey, T.C. and Sotiropoulos, F., Relationship between stirring rate and Reynolds number in the chaotically advected steady flow in a container with exactly counter-rotating lids, Phys. Fluids, 2006, 18, 1-14]. Timedependent regimes are obtained numerically when the value of the Reynolds number is $\operatorname{Re} \geq 1500$. The time dependency is associated with a pulsation of the two vortices found in the steady regime. Experimental visualizations and measurements show that in turbulent regimes the flow also exhibits two vortices, but in this case they travel in the azimuthal direction with a frequency compatible with the frequency obtained in the numerical simulations at much lower Reynolds number. The azimuthal drift of these vortices is associated with the asymmetry of the mean azimuthal flow with respect to the equatorial plane.
\end{abstract}

Keywords: Vortices von Kármán flow; Time-dependent turbulent flow

*Corresponding author. Email: emi@fisfun.uned.es 


\section{Introduction}

Flows in rotating disk systems are not only a subject of fundamental interest as prototype flows for three-dimensional boundary layers but are also a topic of practical importance in the performance improvement of many industrial devices. In turbomachinery, typical flow configurations are the flows between rotating compressor and turbine disks, which can be idealized as flows confined between two coaxial rotating disks. In these two rotating disk flows, the flow supports similarity solutions of von Kármán type. Many research works on these flows are motivated by dynamo experiment feasibility solutions. The dynamo effect in a conducting fluid consists of a self-sustained magnetic field that appears in some configurations (Moffat 1978). The whole problem involves the analysis of momentum, Navier-Stokes equation and the induction equation for the magnetic field $\mathbf{B}$. Because of the physical properties of real materials, a flow that could lead to a dynamo effect has to be in a fully turbulent regime. The experimental approach becomes critical, being one of the key points for the understanding of the flow beneath the dynamo action.

The von Kármán geometry has been proposed as one of those potentially producing a dynamo action. During the last years a great effort has been devoted to the study of this flow (Bourgoin et al. 2002, Pétrélis et al. 2003, Marié et al. 2003, Ravelet et al. 2005) with a successful experiment carried out in 2007 (Monchaux et al. 2007). These experimental flows are performed using propellers instead of flat disks to improve the efficiency (maxima of the velocity flow is compared to the rim velocity). The transition from laminar to turbulent flow has been studied in depth for some propellers (Ravelet et al. 2008).

Nevertheless, some of the results of these experiments (Berhanu et al. 2007) and recent results from some of the authors (de la Torre and Burguete 2007, de la Torre et al. 2007) indicate that the slow scales and the symmetry breaking are important in this instability. It has been shown experimentally (Ravelet et al. 2004) that, for some propellers, this flow can present a global bifurcation from two cells to only one cell that fills the whole container and that is stable in time.

The mechanisms that generate the instabilities strongly depend on the aspect ratio, which is here defined as $\Gamma=$ height $/$ radius $=L / R$, and on the rotation ratio $s=\Omega_{\mathrm{t}} / \Omega_{\mathrm{b}}$ ( $\mathrm{t}$ and $\mathrm{b}$ refer to the top and bottom disks, respectively). The control parameter is the Reynolds number, defined as $\operatorname{Re}=\Omega R^{2} / v$, where $R, \Omega$ and $v$ are the radius of the cylindrical cavity, the rotation rate of one of the end walls and the kinematic viscosity.

In extended cavities, the differential rotation rate between solid boundaries and the incompressible fluid in the core of the cavity create boundary layer-type flows and in the transition process to the turbulence the instabilities arise within the boundary layers (Serre et al. 2001). When the end walls are co-rotating or in slight counter rotation the flow behaves differently to the case of a strong counter rotation rate, so that in the first case the basic flow and the first instability are always axisymmetric and in the second case the destabilization of the basic flow leads to three-dimensional more complex structures. The important role of the rotation ratio, $s$, and the aspect ratio $\Gamma$ on the critical Re and on the mechanisms of the transition has been discussed by Pécheux and Foucault (2006).

In cavities with moderate aspect ratio, $\Gamma \sim \mathrm{O}(1)$ and in the rotor stator configuration, $s=0$, many research work has focused on the study of the vortex breakdown phenomena, which is observed at low Reynolds number (Serre et al. 2002). At higher Reynolds number and $s=0$ the flow is unstable to azimuthal travelling waves 
(Gelfgat et al. 1996, 2001). The case of cavities with $\Gamma \sim \mathrm{O}(1)$, with the end walls in exact counter rotation, $s=-1$, has been studied both experimentally and theoretically by Gelfgat et al. (1996), Nore et al. (2003, 2004, 2005). The base flow is axisymmetric and the instability of the steady flow sets up due to free shear layer instabilities through a Kelvin Helmholtz instability. After the first transition the new state is steady and nonaxisymmetric, the pattern may have different symmetries and several vortices near the equatorial plane, the number of them depends on $\Gamma$. The vortical structures of the threedimensional steady flow have been investigated by Lackey and Sotiropoulos (2006) with a Lagrangian numerical method at $\Gamma=1.5$. This investigation was focused on the steady state, and the stirring rate versus $\mathrm{Re}^{-1 / 2}$ as proposed by Mezićs (Lackey and Sotiropoulos 2006).

In this work, we study the flow in a cylindrical cavity with $\Gamma=2$ when the two end walls are in exact counter rotation, $s=-1$, and the sidewall is at rest. The results of the stability analysis in this configuration, and the investigation of states at low Reynolds numbers, were provided by Nore et al. (2003). At critical Reynolds number $\operatorname{Re}_{c, 1}=349$ the axisymmetric flow becomes unstable to non-axisymmetric perturbations leading to a steady mode called "Mixed mode" (Nore et al. 2003, 2004, 2005). In the range $412 \leq \mathrm{Re} \leq 452$ unsteady states are predicted theoretically and observed experimentally, consisting in a travelling wave and a modulated wave of the previous one. Increasing again the rotation rate the stable solution is found to be the steady "Pure mode", for Re up to 500 by Nore et al. $(2003,2004)$.

This article is focused on the investigation of the time-dependent behavior at high Reynolds numbers. It starts with an outline of the numerical method in section 2 . In section 4, we first present the results of the numerical study, second the results of the experimental study of the turbulent flow and finally the comparison between them. Concluding remarks are given in section 5 .

\section{Mathematical and numerical models}

The governing equations, are the continuity and the Navier-Stokes equations, made dimensionless using $\left[R, \Omega^{-1}, \Omega R\right]$ as units of length, time and of the velocity components. In cylindrical coordinates $(r, \theta, z) \in[0,1] \times[0,2 \pi] \times[0, \Gamma]$, the nondimensional velocity is $\mathbf{u}=(u, v, w)$. The boundary conditions are no-slip for all solid walls, $v= \pm r$ at $z=0$, and $z=\Gamma$ and $\mathbf{u}=0$ at the sidewall $r=1$. The three-dimensional Navier-Stokes equations are solved numerically using a pseudo-spectral numerical method. The coupling between the pressure and the velocity field is treated with a projection method, which uses improved version of the Goda scheme. The time scheme is second-order accurate (Serre and Pulicani 2001). Most of the computations have been performed using a grid with $65 \times 48 \times 65$ collocation points in the radial, azimuthal and axial directions, respectively.

\section{Experimental setup}

The experimental volume consists of a cylindrical vessel made of plexyglass, whose top and bottom ends can rotate independently and are modified at will. The aspect ratio 
$\Gamma=L / R$ can be modified continuously, and for the present results it has been fixed to $\Gamma=2$. In the configuration used for this article, the propellers placed at both ends have 10 curved blades (blades' height, $2 \mathrm{~cm}$ ) that rotate, pushing the fluid with the convex side. The container and propeller radii are, respectively, $R_{\text {cyl }}=10 \mathrm{~cm}$ and $R_{\text {prop }}=8.75 \mathrm{~cm}$. The experimental Reynolds number is defined as $\operatorname{Re}=2 \pi f_{\text {prop }} R_{\text {prop }} R_{\text {cyl }} / \nu$. This definition means that the experimental Re number is slightly lower than the numerical value (about a $12.5 \%, 1-R_{\text {prop }} / R_{\text {cyl }}$ ). The propellers are identical (within a $10 \mu \mathrm{m}$ precision) and there are no asymmetries in the experimental setup. Details of this experimental setup are provided by de la Torre and Burguete (2007).

The geometry of the propellers has been chosen because of two reasons. First, it allows a high efficiency in terms of mean flow velocity compared to rim's velocity. With other geometries, i.e. flat disks, this efficiency can be as low as $1 / 20$, whereas in this configuration it is near 1 . The second reason is that this experiment has been conceived to study the potential application of this flow in a dynamo experiment. For this application, the flow needs a balance between the different velocity components that is achieved using these propellers (de la Torre et al. 2007).

The measurement technique is a classical LDV velocimetry. The propeller velocities have been recorded using different techniques to assure their stability. The data series are, in the best situations, obtained with a data rate of $100 \mathrm{kHz}$ and, to record the slow behavior, we have measured the flow for some hours. These resolutions are large enough to not affect the characterization of the slow time-dependent regimes. The details on the LDV acquisition system are provided by de la Torre and Burguete (2007). The fluctuations are Gaussian and remain below $0.07 \%$ for the short-term times (for records of some hundred turns), and below $0.01 \%$ for long-term acquisitions (for $1 \mathrm{~h}$ or more, compared to the typical turn, $0.1 \mathrm{~s}$ ). With this technique we can record extremely long time series $\left(\sim 10^{5}\right.$ rotation periods) of the velocity field that allow a full characterization of the slow dynamics of the vortices present in the experiments. Using these data the mean flow $\langle u, v, w\rangle$ is obtained averaging the instanteneous flow for at least $10^{2}$ propeller turns.

\section{Results}

\subsection{Numerical results}

Numerical solutions have been obtained in the range $300 \leq \mathrm{Re} \leq 3000$. Spectral numerical simulations are particularly efficient in this range of low Reynolds numbers because they provide well-controlled solutions and only require a moderate number of spectral modes to ensure accurate approximations of the flow quantities. The regimes at $\mathrm{Re} \leq 500$ and intermediate aspect ratio cavities have been investigated by Nore et al. (2003, 2004, 2005) and Lackey and Sotiropoulos (2006). In section 4.1.1 we present the results of the present numerical simulations for $\mathrm{Re} \leq 1000$, including comparisons with previous results in the literature in the same range of parameters. In section 4.1.2, we present the features of the numerical results which are useful to analyze the timedependent solutions at higher $\mathrm{Re}: \mathrm{Re}=1500$ and 3000 . 
4.1.1. Steady states. In the basic steady state the fluid rotates with the end walls and is expelled radially by the centrifugal effect. To ensure mass conservation the fluid is pumped along the axis of the cylinder toward the end walls and recirculates near the cylinder wall involving two symmetric toric cells with respect to an azimuthal shear layer at mid-height. Our computations for $\mathrm{Re}=300$ are in full agreement with the basic flow obtained by Nore et al. (2003) and the velocity maxima agree with the values provided in that reference.

According to the stability analysis results of Nore et al. (2003), the critical Reynolds number for the onset of $3 \mathrm{D}$ flow instability for an aspect ratio $\Gamma=2$ considered herein is $\operatorname{Re}_{c, 1}=349$.

We performed numerical computations of the flow at $\operatorname{Re}>\operatorname{Re}_{c, 1}$ using different initial conditions and the results show that in order to obtain $3 \mathrm{D}$ solutions it is necessary to have a finite non-axisymmetric perturbation as initial condition, as was already pointed out by Lackey and Sotiropoulos (2006) in the case of a cavity with $\Gamma=1$. This transition was found to be subcritical since the amplitude of the perturbations is small (typically $0.1 \%$ ) the perturbations decay and when the amplitude is larger, typically $1 \%$, the flow evolves to a $3 \mathrm{D}$ flow.

Thus, for $\mathrm{Re}=355$ the axisymmetric solution is stable to infinitesimal perturbations. When it is perturbed adequately, the flow becomes 3D and it has the Fourier azimuthal components $m=0$ and $m=1$. The perturbations with other azimuthal components $m>1$ are smaller and decrease in time. The evolution in time of the perturbations with $m=1,2,3$ and 4 azimuthal modes is represented in figure 1 (a). At $\operatorname{Re}=440$ the 3D flow is different from the $3 \mathrm{D}$ solution obtained at $\mathrm{Re}=355$ and the evolution in time of the Fourier amplitudes $m=1,2,3,4$ are shown in figure 1(a). In this case the asymmetric solution has azimuthal components $m=0$ and 2, and the evolution of the amplitudes is represented in figure $1(\mathrm{~b})$.

In figure 2, the perturbations of the axisymmetric velocity fields are represented at $\mathrm{Re}=390$ (right) and 440 (left) in the meridian and equatorial planes. In these plots the $m=1$ and $m=2$ components of the flow have been obtained by subtracting the $m=0$ component from the steady numerical solutions.

The qualitative difference between the spatial patterns of both steady states, which were already observed by Nore et al. (2003), is also shown in figure 3 where the isoazimuthal velocity is represented for $\mathrm{Re}=390$ and 440 . These graphics are pictures in perspective from the top of the cavity. There are two and four darker regions, respectively. The two darker regions in (a) represent a maximum and a minimum of the azimuthal velocity that correspond to an "one cat's eye pattern" and the four darker regions in the second graphics (b) represent two maxima and two minima, corresponding to a "two cat's eye pattern". In the present numerical computations the Reynolds number was increased up to $\mathrm{Re}=1000$. The resulting flow is steady and it does not present any qualitative difference with respect to the flow obtained at $\operatorname{Re}=440$. Therefore the steady solution presents a "two cat's eye pattern" similar to the patterns at lower Re. The total computation time for $\mathrm{Re}=1000$ was $t=400$.

4.1.2. Time-dependent flow. The characteristic time scales in this system, like in other rotating fluids, are the rotation time, $\Omega^{-1}$ which is typically the time for the development of the viscous boundary layer during spin up, the Ekman time, $R /(\Omega v)^{1 / 2}$, which represents the time required for the secondary flow to move a fluid particle along 

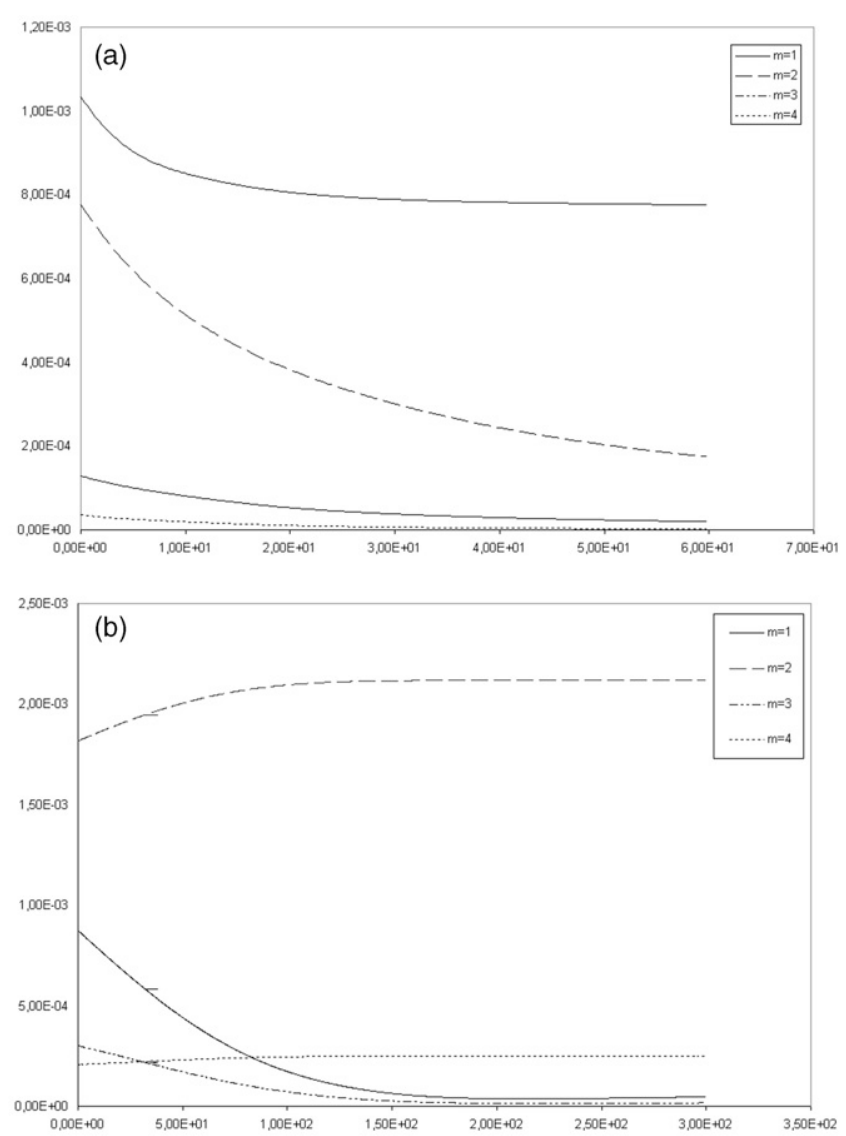

Figure 1. Temporal evolution of the azimuthal amplitudes of the $m=1,2,3$ and 4 modes at $\operatorname{Re}=355$ (a) and 440 (b). The amplitude of $m=0$ is about $2 \times 10^{-2}$ in both cases.

the characteristic length, $R$, and the viscous diffusion time $R^{2} / \nu$, which represents the damping time for the residual oscillations.

The dimensionless rotation period is $2 \pi$, the dimensionless Ekman time is $\tau_{E k}=\operatorname{Re}^{1 / 2}$, and the dimensionless viscous diffusion time is $\tau_{v}=\mathrm{Re}$. In order to interpret the results the frequencies of the time-dependent flow and the time of computations can be referred to these values.

Two time-dependent flows have been investigated, at $\mathrm{Re}=1500$ and $\mathrm{Re}=3000$, corresponding to an oscillatory and an aperiodic regimes. Time dependent flows have been predicted in a very narrow gap of low Reynolds numbers, $412 \leq \operatorname{Re} \leq 452$ by Nore et al. (2005). As these time-dependent states were not the objective of the present work we did not perform an exhaustive numerical search of theses states. Only some damped oscillations were observed for $\mathrm{Re}=420$.

4.1.3. Flow at $\operatorname{Re}=1500$. At $\mathrm{Re}=1500$ the time dependence of the numerical solution is oscillatory. The frequency is 0.21 times the rotation frequency, which corresponds to 


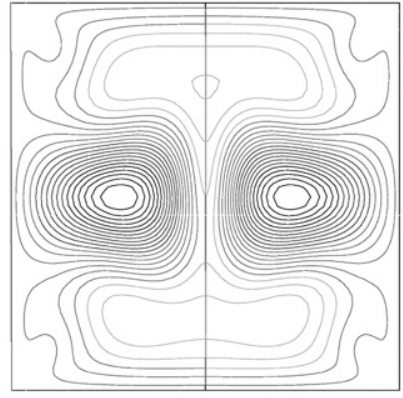

$\mathrm{m}=1, \mathrm{Re}=390$

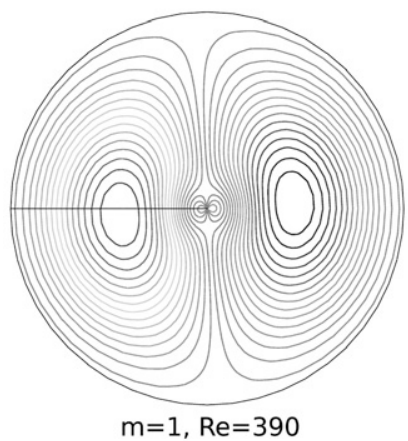

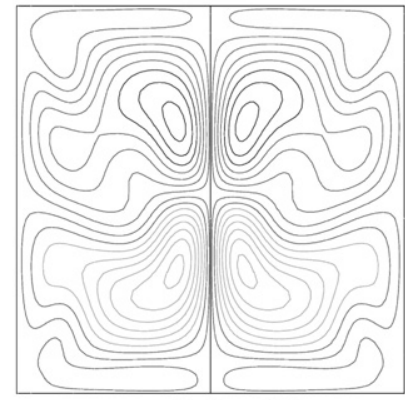

$m=2, R e=440$

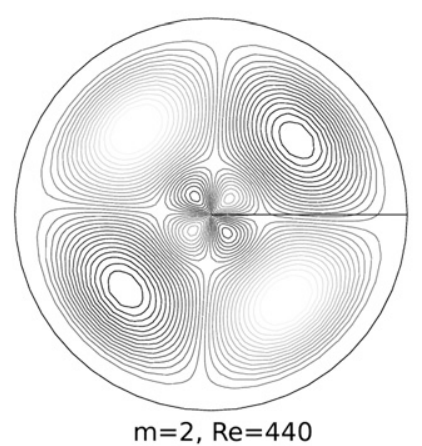

Figure 2. Iso lines of the azimuthal velocity perturbations of the flow at $\mathrm{Re}=390$ and 440 in the meridian planes $\theta=0, \pi$ and in the equatorial plane $z=\Gamma / 2$. The amplitude of the axisymmetric $m=0$ mode has been subtracted from the numerical flow in both figures.

a value of the period $T /(2 \pi)=4.8$. The relative amplitude of the Fourier modes is similar to the solution at $\mathrm{Re}=500$ and 1000 . Only even modes are present in the flow: the amplitudes of $m=2$ and 4 modes are one order of magnitude smaller than the amplitude of $m=0$, and the amplitude of the $m=6$ mode is two orders of magnitude smaller. The amplitudes of odd modes are smaller still. In the velocity field there are two vortices similar to those exhibited in figure 3(b) corresponding to the two cat's eyes pattern. These vortices remain at the same position and the oscillation in time corresponds to periodic fluctuations in time of the vortices intensity. The origin of this frequency is not the instability of the boundary layers near the disks, because it is not observed in the graphics, and because as the frequency of the boundary layer instability is about 7-12 times the rotation frequency it is very different from the frequency obtained in these computations.

The frequency of the oscillations obtained here is compatible with the oscillation of the axisymmetric $m=0$ azimuthal mode. In order to illustrate this connection, we have included in table 1 the critical parameters of the first unstable modes in a counter rotating cavity, $s=-1$, together with the results in a rotor-stator configuration, $s=0$. It is well established that, when the end walls are in exact counter rotation, the first instability is to a non-axisymmetric steady state. The values of the linear thresholds of axisymmetric modes and other non-axisymmetric modes provided in the literature have been presented in table 1 . When the end walls are in rotor-stator configuration the first instability is to an oscillatory axisymmetric state. Although the critical parameters are valid only near the critical conditions, in principle, further transitions are based on the 

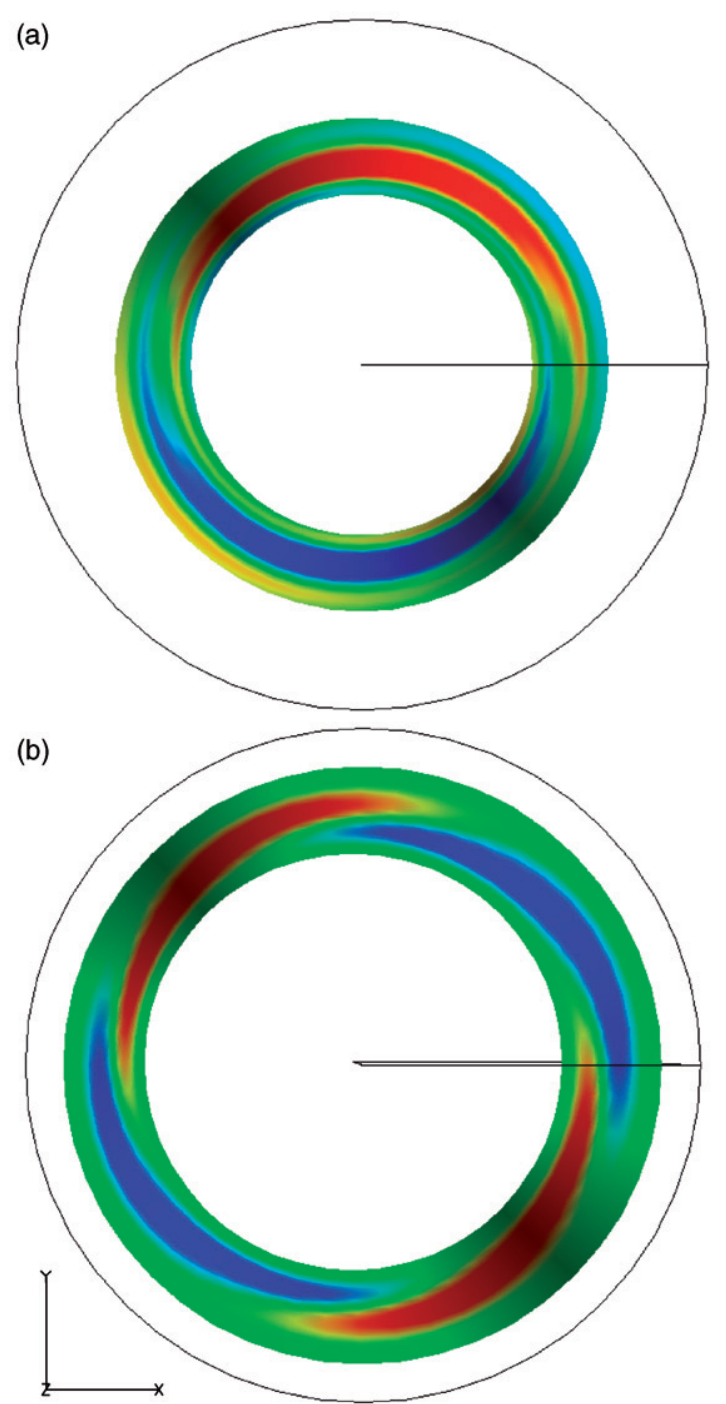

Figure 3. View from above of azimuthal velocity iso surfaces showing a maximum and a minimum (i.e. one cat's eye pattern) at $r=0.5$ for $\mathrm{Re}=390$ (a) and two maxima and two minima (two cat's eyes) at $r=0.75$ for $\operatorname{Re}=440$ (b).

bifurcation diagrams and in this context we compare the frequencies of axisymmetric mode $m=0$ obtained numerically $\omega=0.21$ with the predictions of linear stability analysis, 0.3 , (table 1). But the origin of these two behaviors might not be the same.

4.1.4. Flow at $\operatorname{Re}=\mathbf{3 0 0 0}$. When the rotation rate is increased up to $\mathrm{Re}=3000$ the time signal is aperiodic. The instantaneous pictures of the flow (figure 4) exhibit a two cat's eyes pattern and the azimuthal Fourier amplitudes reveal that the even azimuthal modes predominate, similarly to the features of the solution for $\mathrm{Re}=1500$. Looking at the time evolution of the flow pattern, it is observed that the time-dependent behavior 
Table 1. Thresholds and critical frequencies for azimuthal modes $m=0-3$ for a rotor-stator flow $s=0$ and counter rotating flow, $s=-1$.

\begin{tabular}{lcccc}
\hline$(s, \Gamma)$ & $m$ & $\operatorname{Re}$ & $\omega$ & $\frac{T}{2 \pi}$ \\
\hline$(-1,2)^{\mathrm{a}}$ & 0 & 1852 & 0.3 & 3.3 \\
& 1 & 349 & 0 & 0 \\
& 2 & 401 & 0 & 0 \\
$(-1,1.5)^{\mathrm{b}}$ & 3 & 625 & 0 & 0 \\
& 0 & 1644 & 0.284 & 3.52 \\
$(0,2)^{\mathrm{c}}$ & 1 & 332 & 0 & 0 \\
& 0 & $2.58 \times 10^{3}$ & 0.243 & 4.12 \\
& 1 & $3.3 \times 10^{3}$ & 0.07 & 14.3 \\
& 2 & $3.0 \times 10^{3}$ & -0.015 & 69.2 \\
$(0,1.5)^{\mathrm{d}}$ & 3 & $3.92 \times 10^{3}$ & -0.105 & 9.54 \\
& 0 & $2.7 \times 10^{3}$ & 0.24 & 4.22 \\
& 1 & $3.2 \times 10^{3}$ & $\simeq 0.5$ & 2 \\
& 3 & $2.5 \times 10^{3}$ & $\simeq 0$ & 0 \\
& $3.1 \times 10^{3}$ & $\simeq 0.11$ & 9.09 \\
\hline
\end{tabular}

Note: ${ }^{\mathrm{a}}$ Nore et al. $(2003,2004),{ }^{\mathrm{b}}$ Gelfgat et al. (1996), ${ }^{\mathrm{c}}$ Gelfgat et al. (2001), ${ }^{\mathrm{d}}$ Gelfgat et al. (2001) and Nore et al. (2004).

of the variables is chaotic and that the vortices do no travel. Differently from the pattern obtained at $\operatorname{Re}=1500$, where the vortices do not move, in this case the center of each vortex moves around a steady position, remaining close to it and without a fixed trajectory.

The velocity field $(v, w)$ in the plane $(\theta, z)$ at $r=0.9$ is represented at several time steps with $\Delta t \approx 30$ in figure 4 . The graphics represent the evolution of the flow over a long time, $t=300$. In the chaotic signal we have observed that the amplitude of the $m=4$ is greater than that of $m=2$ in some short periods of time. Correspondingly, in most instantaneous graphics there are two visible vortices and in some time steps the velocity plots display four vortices, showing the relevance of the $m=4$ mode in the spatial azimuthal spectrum. The contribution of even modes continues to be small: the amplitudes of the odd modes are at least two orders of magnitude smaller than those of the even modes. Some indicative amplitudes are: $2 \times 10^{-2}$ the magnitude of $m=0$ and $\mathrm{O}\left(10^{-3}\right)$ the magnitude of $m=2,4$ and $m=6$. As we pointed before the relative magnitude of $m=2$ and $m=4$ varies, being greater or smaller in different instants.

The three-dimensional pattern flow is complex. Figure 5 represents the instantaneous velocity field projected on meridian planes: $\theta=0, \pi / 12, \pi / 6$ in the first row from left to right, and $\theta=\pi / 4, \pi / 3$ and $5 \pi / 12$ in the second row and from left to right. Due to the symmetry exhibited by the solution, the patterns in the other half cylinder $[\pi / 2, \pi]$ are alike. In all the graphics the recirculation of the flow can be observed: near the axis of rotation the flow goes toward the end walls, near the end walls there are boundary layers and the flow travels to the sidewall, then it goes to the equatorial plane along the sidewall, and recirculates from the sidewall toward the axis near to the equatorial plane $z \approx 0$.

\subsection{Experimental results}

For $\operatorname{Re}<10^{4}$ the mean flow is almost symmetric with respect to the equatorial plane and consequently the azimuthal velocity is nearly null in this plane. As the rotation rate 

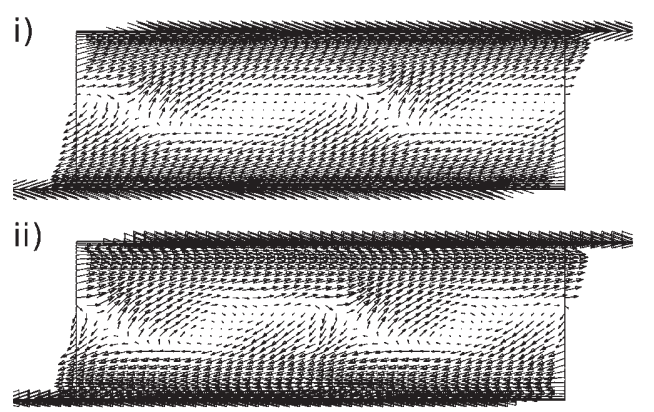

iii)

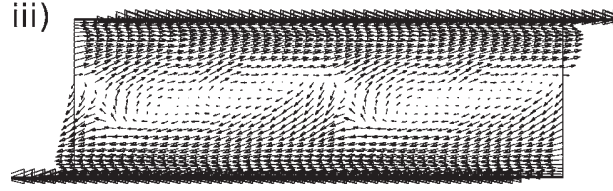

iv)

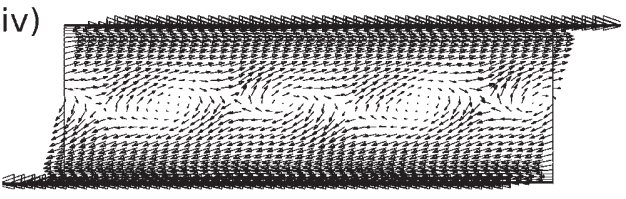

v)

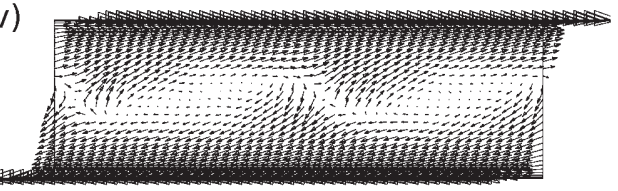

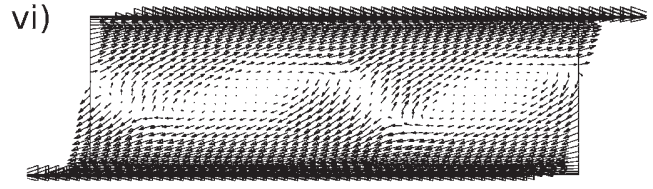
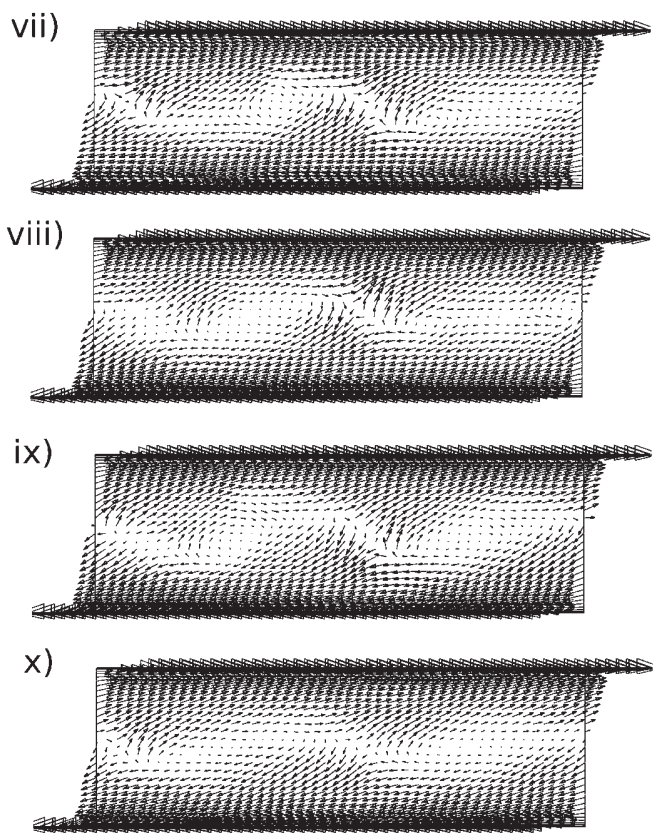

Figure 4. Instantaneous iso-plots of the $(v, w)$ components of the velocity field at $\mathrm{Re}=3000$ in a $(\theta, z)$ plane at $r=0.9$.

is increased it is observed a dissymmetry, which becomes more notorious, until an asymptotic state is reached for $\mathrm{Re}=10^{5}$. The asymmetric flow has two vortices at opposite azimuth angles, with a characteristic size $D_{\text {vortex }} \approx 5 \mathrm{~cm}$ which appear simultaneously to the asymmetry of the mean flow. The stream vectors and contour plots of $v$ are represented in the figure 6 . The contour plot of azimuthal velocity $v$ and the velocity field $(u, w)$ projected on the meridian plane $\theta=0$, is represented when $\mathrm{Re}=3 \times 10^{5}$. There are two toroidal cells, each of them near one the propellers. Near the upper and lower ends the flow is aspired through the axis toward the propellers, as it was observed in the numerical results. The vortices move with a precessing movement with a positive or negative velocity in the equatorial plane (azimuthal velocity). This displacement defines two different states labelled $N$ or $S$ depending on whether the vortices follow the up or bottom propeller.

The asymmetric states can be characterized using different variables. One is the position of the frontier $z_{0}$, defined as the $z$ position where the azimuthal velocity is zero, $v=0$. When the $N$ (resp. $S$ ) state dominates, $z_{0}$ is below (resp. above) the equator. In figure 6 , the $N$ state dominates, being $z_{0} \sim-0.15$. Another possibility is measuring $v_{\text {eq }}$, the mean azimuthal velocity at an equatorial point near the wall $(r \approx 0.9$ with the LDV system) that reflects the vortices velocity. This mean velocity 

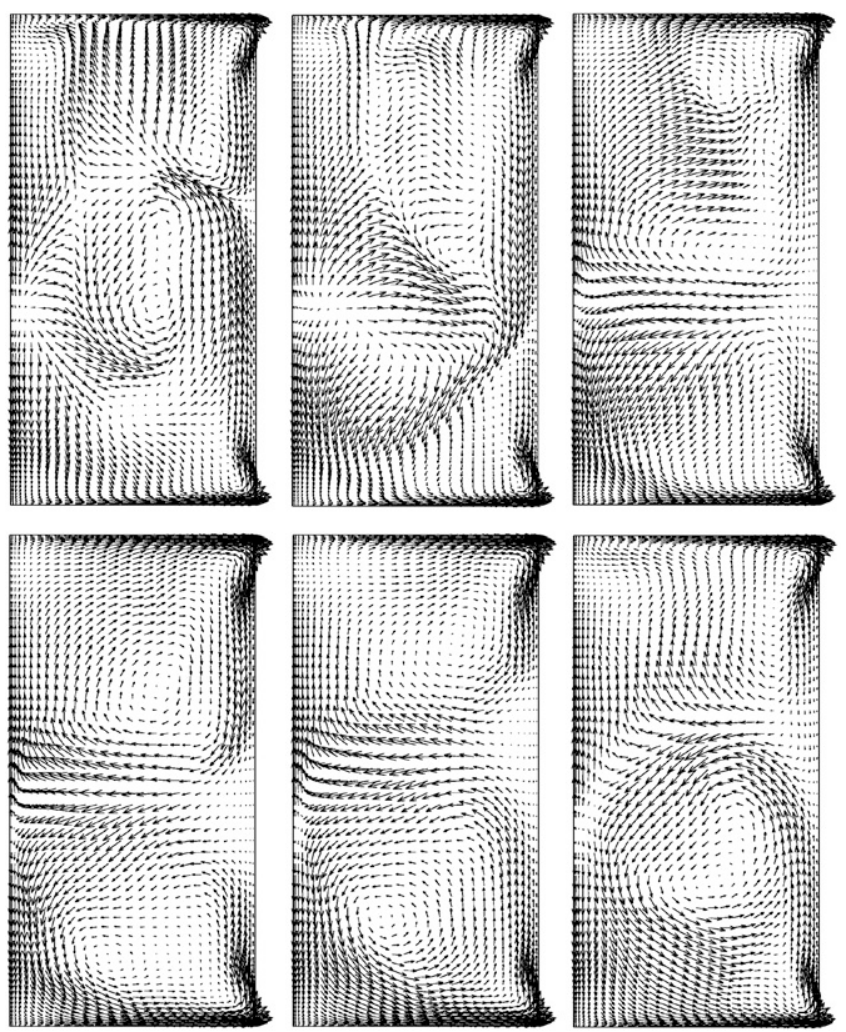

Figure 5. Instantaneous velocity field projected on the first quadrant meridian planes $\theta=0, \pi / 12, \pi / 6$, and in the next line on the second quadrant planes $\theta=0, \pi / 4,0, \pi / 3,5 \pi / 12$ at $\operatorname{Re}=3000$.
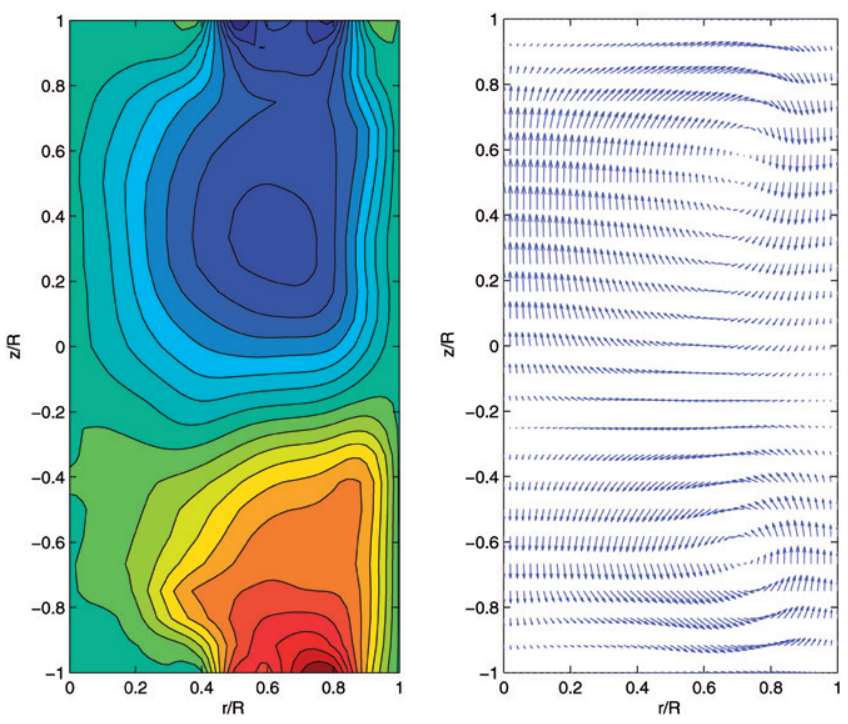

Figure 6. Turbulent flow at $\operatorname{Re}=10^{5}$ averaged in $\theta$ : at left it is represented a contour plot of the azimuthal velocity (toroidal velocity) and at right the $(u, w)$ velocity field in the same plane $\theta=0$. 
(a)

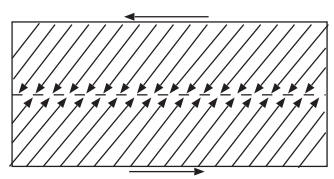

Mean flow

(b)

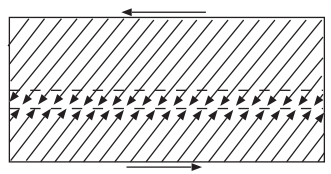

Mean flow

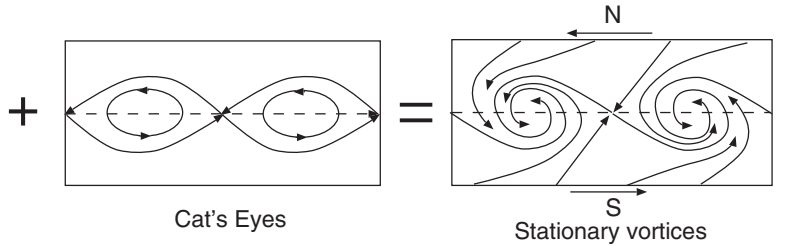

Stationary vortices

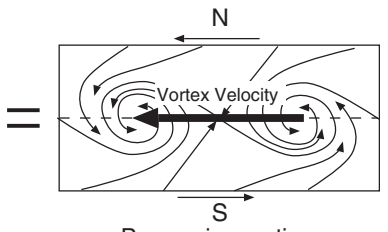

Precessing vortices

Figure 7. Scheme in a $(\theta, z)$ plane of the cat' eyes behavior when the flow is symmetric (a) and not symmetric (b) with respect to the equatorial plane, $z=\Gamma / 2$.

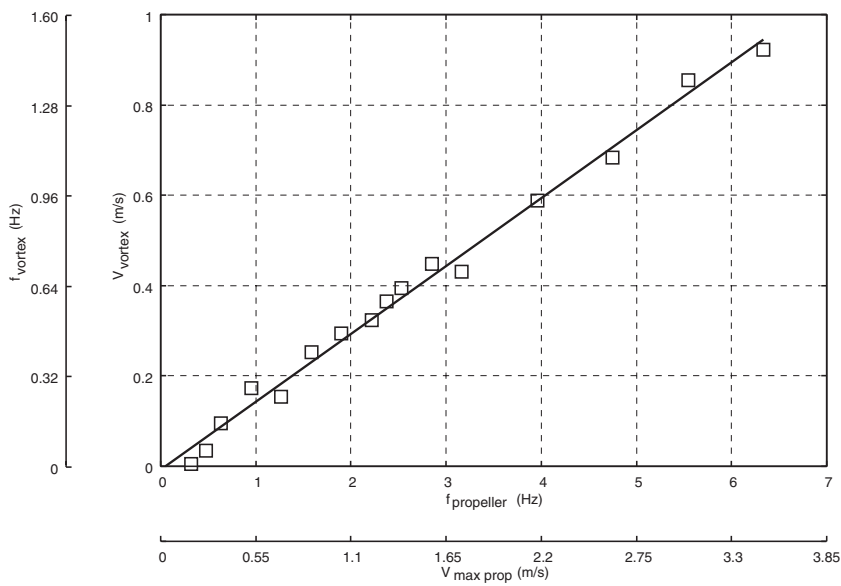

Figure 8. Azimuthal velocity as a function of the rotation velocity.

appears because of the displacement of the shear layer (around $\pm \Gamma / 20$ ) from the equator, breaking the symmetry with respect to the equatorial plane (figure 7), and remains stable in time (for intermediate acquisition times, i.e. around one thousand propeller periods) and proportional to the propellers rim velocity (figure 8). Nevertheless these states are not completely stable, because rare spontaneous jumps (inversions) between $N$ and $S$ states can appear for very long acquisition times. These inversions were observed in a range of $10^{4}<\mathrm{Re}<10^{5}$. The analysis of the experimental data shows that the time of residence, i.e. the time that the system stays in one of the states, follows a law $\rho(t)=1 / T_{0} \exp \left(-t / T_{0}\right)$, where the characteristic time is $T_{0}=1484 s=7020 \times 2 \pi / \Omega=0.2 \tau_{v}$, being $\tau_{v}$ the diffusion time scale (de la Torre et al. 2007).

Experimental results have been performed with rotation rates slightly different from $s=\Omega_{\mathrm{up}} / \Omega_{\text {low }}=-1$. When the propellers are not exactly counter rotating, no inversions 

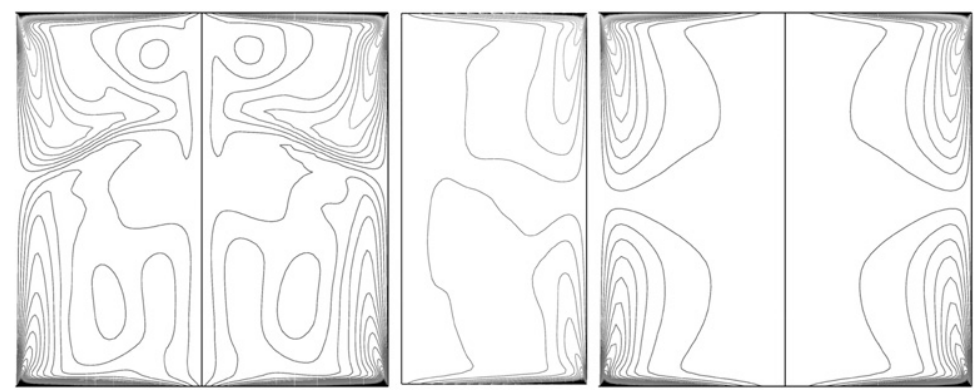

Figure 9. Numerical flow at $\operatorname{Re}=3000$. Azimuthal velocity instantaneous (left) averaged in $\theta$ (center) and averaged in $\theta$ and in time (right) $\langle v\rangle_{\theta, t}$, in planes $\theta=0$ and $\theta=(0, \pi)$.

have been observed when $|s+1|>0.001$. A very narrow hysteresis window has been found for $|s+1|<0.001$.

\subsection{Comparison between experimental and numerical results}

In the experimental visualizations the cat's eyes pattern precesses, travelling in the azimuthal direction. The velocity of the vortices is represented as a function of the velocity of the propeller in figure 8 . The time frequency is 0.24 times the frequency of the propellers, corresponding to a period $T /(2 \pi)=4.2$. In the numerical computation the frequency is 0.21 times the rotation frequency of the end walls. The cat's eyes pattern oscillates about a standing position. These values of the frequency fall into the range of values found for the oscillation of the $m=0$ mode, as it is seen in table 1 .

These different behaviors may be explained by observing the averaged flow. When the mean flow averaged in the azimuthal direction $\langle\mathbf{u}\rangle_{\theta, t}$ is symmetric with respect to the equatorial plane, the vortices are placed near the equator, the averaged azimuthal flow is zero in that plane and the vortices do not precess as it is represented in the scheme of figure 7(a). Similarly an averaged asymmetric flow precesses as it is represented in the same scheme in 7(b): when the flow is asymmetric there is a net drift velocity in the direction of the azimuthal velocity at the equator. In figure 7(b) it is represented a drift flow to the left. The averaged turbulent flow at $\mathrm{Re}=3 \times 10^{5}$ presented in figure 6 exhibits the asymmetry with respect to the $z=\Gamma / 2$ plane, corresponding to a flow with the same characteristics that in figure 7(b).

The experimental pattern in figure 6(b) may be compared with the averaged velocity field of the numerical solution at $\mathrm{Re}=3000$, in figure 9 . In the numerical solution the nuclei of the tori are close to the sidewall and in the experimental flow, due to the effect of the propellers and to the different (higher) Reynolds number, the circulation zones appear for more intermediate radii.

At $\mathrm{Re}=3000$ the numerical flow in figure 5 is not symmetric with respect to the equatorial plane, $z=\Gamma / 2$, in every $\theta$ plane but the velocity field averaged in time and in $\theta$ is symmetric. When the instantaneous flow is averaged over all the $\theta$-planes, i.e. $\langle u\rangle_{\theta}$, $\langle v\rangle_{\theta},\langle w\rangle_{\theta}$ the flow is almost symmetric, as it is shown in figure 9 . In the equatorial plane, the azimuthal velocity is nearly zero and the situation corresponds to figure $7(\mathrm{a})$. 
However, because of the differences in the configuration in both analyzed systems, a whole agreement can not be expected.

\section{Concluding remarks}

The characteristics of the time-dependent swirling flows have been investigated in a $\Gamma=2$ cylindrical cavity with counter rotating end walls. Spectral direct numerical simulations have been performed up to $\mathrm{Re}=3000$ and turbulent regimes have been investigated experimentally for Reynolds numbers from $10^{3}$ to $10^{6}$. The numerical base flow is steady and axisymmetric. Increasing the rotation rate of the end walls the flow bifurcates first to a steady 3D state with $m=0$ and 1 azimuthal modes (see the flow at $\mathrm{Re}=355$ ) and then to a steady state with $m=0,2$ and 4 azimuthal modes (see the flow at $\operatorname{Re}=400)$.

Time-dependent states have been investigated at $\mathrm{Re}=1500$ and $\mathrm{Re}=3000$ corresponding to an oscillatory and a chaotic state, respectively. In both flows only the first even modes remain significant, as it was observed in the steady regimes at lower Reynolds numbers. The oscillatory behavior at $\mathrm{Re}=1500$ corresponds to an oscillatory variation in the intensity of the two cat's eyes. The value of the frequency, equal to 0.21 times the frequency of the rotating end walls, is compatible with the values obtained in the linear stability predictions for the instability of the $m=0$ mode, i.e. the instability of the axisymmetric steady state to an axisymmetric oscillatory flow. At $\mathrm{Re}=3000$ the aperiodic flow is characterized by the motion of every cat's eye centering around a steady position, remaining close to it and without a fixed trajectory.

Experiments performed in turbulent regimes have shown that these vortical patterns remain qualitatively alike over a large range of Reynolds numbers. Nevertheless, in the fully turbulent flow the vortices travel with a drift azimuthal velocity and a frequency equal to 0.24 times the frequency of the propellers. These values remain compatible with the frequency obtained in the numerical simulations at much lower Reynolds numbers. The azimuthal drift of these vortices is associated to the symmetry breaking in the mean azimuthal flow with respect to the equatorial plane which was not numerically observed in the laminar regime.

\section{Acknowledgements}

We acknowledge financial support from Spanish government through contract numbers FIS2004-06596, FIS2007-66004, FIS2008-01126 and MTM2007-62482. A. de la Torre thanks the Asociación de Amigos for a grant from the University of Navarra.

\section{References}

Berhanu, M., Monchaux, R., Fauve, S., Mordant, N., Pétrélis, F., Chiffaudel, A., Daviaud, F., Dubrulle, B., Marié, L., Ravelet, F., Bourgoin, M., Odier, P., Pinton, J. F. and Volk, R., Magnetic field reversals in an experimental turbulent dynamo. Europhys. Lett., 2007, 77, 59001. 
Bourgoin, M., Marié, L. and Petrelis, J.B.F., Magnetohydrodynamics measurements in the von Kármán sodium experiment. Phys. Fluids, 2002, 14, 3046-3058.

de la Torre, A. and Burguete, J., Slow dynamics in a turbulent von Kármán swirling flow. Phys. Rev. Lett., 2007, 99, 1-3.

de la Torre, A., Burguete, J. and Pérez-García, C., Influence of time-dependent flows on the threshold of the kinematic dynamo action. Eur. Phys. J. ST, 2007, 146, 313-320.

Gelfgat, A.Y., Bar-Yoseph, P.Z. and Solan, A., Steady states and oscillatory instability of swirling flow in a cylinder with rotating top and bottom. Phys. Fluids, 1996, 8, 2614-2625.

Gelfgat, A.Y., Bar-Yoseph, P.Z. and Solan, A., Three-dimensional instability of axisymmetric flow in a rotating lid-cylinder enclosure. J. Fluid Mech., 2001, 438, 363-377.

Lackey, T.C. and Sotiropoulos, F., Relationship between stirring rate and Reynolds number in the chaotically advected steady flow in a container with exactly counter-rotating lids. Phys. Fluids, 2006, 18, $1-14$.

Marié, L., Burguete, J., Daviaud, F. and Léorat, J., Numerical study of homogeneous dynamo based on experimental von Kármán type flows. Eur. J. Phys. B, 2003, 33, 469-485.

Moffat, H.K., Magnetic Field Generation in Electrically Conducting Fluids, 1978 (Cambridge, UK: Cambridge Monographs on Mechanics, Cambridge University Press).

Monchaux, R., Berhanu, M., Bourgoin, M., Moulin, M., Odier, P., Pinton, J. F., Volk, R., Fauve, S., Mordant, N., Petrelis, F., Chiffaudel, A., Daviaud, F., Dubrulle, B., Gasquet, C., Marié, L. and Ravelet, F., Generation of a magnetic field by dynamo action in a turbulent flow of liquid sodium. Phys. Rev. Lett., 2007, 98, 044502.

Nore, C., Moisy, F. and Quartier, L., Experimental observation of near-heteroclinic cycles in the von Kármán swirling flow. Phys. Fluids, 2005, 17, 064103.

Nore, C., Tartar, M., Daube, O. and Tuckerman, L.S., Survey of instability thresholds of flow between exactly counter-rotating disks. J. Fluid Mech., 2004, 511, 45-65.

Nore, C., Tuckerman, L.S., Daube, O. and Xin, S., The 1:2 mode interaction in exactly counter-rotating von Kármán swirling flow. J. Fluid Mech., 2003, 477, 51-88.

Pécheux, J. and Foucault, E., Axisymmetric instabilities between coaxial rotating disks. J. Fluid Mech., 2006, 563, 293-318.

Pétrélis, F., Bourgoin, M., Marié, L., Burguete, J., Chiffaudel, A., Daviaud, F., Fauve, S., Odier, P. and Pinton, J.-F., Nonlinear magnetic induction by helical motion in a liquid sodium turbulent flow. Phys. Rev. Letters, 2003, 90, 501-3.

Ravelet, A., Chiffaudel, A. and Daviaud, F., Supercritical transition to turbulence in an inertially driven von Kármán closed flow. J. Fluid Mech., 2008, 601, 339-364.

Ravelet, F., Chiffaudel, A., Daviaud, F. and Leorat, J., Toward an experimental von Kármán dynamo: Numerical studies for an optimized design. Phys. Fluids, 2005, 17, 117104.

Ravelet, F., Marié, L., Chiffaudel, A. and Daviaud, F., Multistability and memory effect in a highly turbulent flow: experimental evidence for a global bifurcation. Phys. Rev. Lett., 2004, 6, 164501.

Serre, E. and Bontoux, P., Vortex breakdown in a three-dimensional swirling flow. J. Fluid Mech., 2002, 459, 347-370.

Serre, E., Crespo del Arco, E. and Bontoux, P., Annular and spiral patterns in flows between rotating and stationary discs. J. Fluid Mech., 2001, 420, 1-36.

Serre, E. and Pulicani, J.P., A three-dimensional pseudospectral method for rotating flows in a cylinder. Comp. Fluids, 2001, 30, 491-519. 